\title{
UV spectroscopy of the hybrid PG 1159-type central stars of the planetary nebulae NGC 7094 and Abell 43
}

\author{
M. Ziegler, ${ }^{1}$ T. Rauch, ${ }^{1}$ K. Werner, ${ }^{1}$ L. Koesterke, ${ }^{2}$ and J. W. Kruk ${ }^{3}$ \\ ${ }^{1}$ Institute for Astronomy and Astrophysics, Kepler Center for Astro and Particle Physics, \\ Eberhard Karls University, Tübingen, Germany \\ 2 Texas Advanced Computing Center, University of Texas, Austin, U.S.A. \\ ${ }^{3}$ Department of Physics and Astronomy, Johns Hopkins University, Baltimore, U.S.A.
}

\begin{abstract}
Hydrogen-deficient post-AGB stars have experienced a late helium-shell flash that mixes the hydrogen-rich envelope and the helium-rich intershell. The amount of hydrogen remaining in the stellar envelope depends on the particular moment when this late thermal pulse occurs.

Previous spectral analyses of hydrogen-deficient post-AGB stars revealed strong iron deficiencies of up to 1 dex. A possible explanation may be neutron captures due to an efficient s-process on the AGB that transformed iron into heavier elements. An enhanced nickel abundance would, thus, be an indication for this scenario.

We performed a detailed spectral analysis by means of NLTE model-atmosphere techniques based on high-resolution UV observations of the two PG 1159-type central stars of the planetary nebulae NGC 7094 and Abell 43 which are spectroscopic twins, i.e. they exhibit very similar spectra. We confirmed a strong iron-deficiency of at least one dex in both stars. The search for nickel lines in their UV spectra was entirely negative. We find that both stars are also nickel-deficient by at least one dex.
\end{abstract}

Individual Objects: NGC 7094, Abell 43

\section{Introduction}

NGC 7094 and Abell 43 are so-called hybrid-PG 1159 type central stars of planetary nebulae. In contrast to other PG 1159 stars they exhibit hydrogen lines in their spectra. Hybrid-PG 1159 stars experience a final thermal pulse (TP) while they are still on the asymptotic giant branch (AGB) and hydrogen-shell burning is still "on" (cf. Herwig 2001). Hydrogen is then diluted to $\sim 17 \%$ by mass.

In a previous spectral analysis, Dreizler et al. (1997) found $T_{\text {eff }}=110, \log g=5.7$, and $\mathrm{H}: \mathrm{He}=36: 43$ (by mass) for both stars, NGC 7094 and Abell 43. A determination of the iron abundance of NGC 7094 revealed a strong deficiency of at least one dex (Miksa et al. 2002). Neutron capture due to the s-process in the former AGB star could have transformed Fe into heavier nuclei.

At the relevant temperature regime, all elements are highly ionized. The dominant ionization stages of the iron-group elements in the line-forming regions are VI and VII. Lines of these are located in the UV and FUV range, which were only accessible by the Far UItraviolet Spectroscopic Explorer (FUSE) and the Hubble Space Telescope (HST) with STIS (Space Telescope Imaging Spectrograph). To determine the iron and nickel abundances of 
NGC 7094 and Abell 43, we analyzed high-resolution and high S/N observations obtained with these spectrographs.

\section{Spectral analysis}

Since the spectral analysis of NGC 7094 and Abell 43 by Dreizler et al. (1997) using low resolution TWIN spectra, our model atmospheres as well as the available observations have been improved (see Rauch \& Deetjen 2003, Werner et al. 2003 for details). Based on a high-resolution $(R=18000)$, high-S/N optical spectrum (obtained during the SPY campaign, Napiwotzki et al. 2001) and theoretical spectral energy distributions (SEDs) provided by $T h e o S S A^{1}$, a service by $G A V O^{2}$, we found that we achieve a much better fit with the observation at $T_{\text {eff }}=100 \pm 10 \mathrm{kK}, \log g=5.0 \pm 0.3$, and $\mathrm{H}: \mathrm{He}=9: 79( \pm 0.5 \mathrm{dex})$. Due to a lower $\mathrm{S} / \mathrm{N}$, the error ranges are a factor of two larger for Abell 43.

We adopted these parameters and calculated plane-parallel, hydrostatic NLTE model atmospheres using the Tübingen Model Atmosphere Package (TMAP), which considered the opacities of 23 elements $(\mathrm{H}, \mathrm{He}, \mathrm{C}, \mathrm{N}, \mathrm{O}, \mathrm{F}, \mathrm{Ne}, \mathrm{Na}, \mathrm{Mg}, \mathrm{Al}, \mathrm{Si}, \mathrm{P}, \mathrm{S}, \mathrm{Ar}, \mathrm{Ca}, \mathrm{Sc}, \mathrm{Ti}$, $\mathrm{V}, \mathrm{Cr}, \mathrm{Mn}, \mathrm{Fe}, \mathrm{Co}$, and $\mathrm{Ni}$ ). The abundances of $\mathrm{C}, \mathrm{N}, \mathrm{O}$, and $\mathrm{Si}$ could be determined $([X]=1.7,-0.9,-0.2,0.4$, respectively; $[X]$ denotes log (abundance / solar abundance) of species $X$ ). For the other elements, only upper limits could be determined (for details, see Ziegler et al. in prep.). The subsolar oxygen abundance is unexplained because carbon as well as oxygen should be dredged up during the thermal pulse and therefore be enhanced.

Our analysis of NGC 7094 and Abell 43 confirmed the strong Fe-deficiency that was previously found (Miksa et al. 2002). Due to the lack of reliable atomic data for post-iron elements and the expected high ionization stages, we were able to search for cobalt and nickel lines only. Although we have derived new parameter values which improved the agreement between model and observation, we were not able to identify any $\mathrm{Co}$ or Ni line. Test calculations show that these lines should be visible at $[\mathrm{Co}]=0$ and $[\mathrm{Ni}]=-1$. While an increased $\mathrm{Ni}$ abundance would be a clear evidence for a transformation of $\mathrm{Fe}$ into $\mathrm{Ni}$ by neutron captures, the $\mathrm{Ni}$ deficiency may be an indication that the s-process transformed $\mathrm{Ni}$ into even heavier elements. Due to the lack of suitable atomic data at the investigated parameter regime, the analysis of abundances of trans-iron group elements is presently impossible.

\section{Results and conclusions}

Our analysis of the two hybrid PG 1159 stars NGC 7094 and Abell 43 confirmed the previously found Fe-deficiency of at least one dex. Although we have derived new parameter values which improved the agreement between model and observation, we were not able to find any $\mathrm{Ni}$ enhancement. Instead, we found $\mathrm{Ni}$ to be deficient by at least one dex. It is therefore possible that the s-process also converted $\mathrm{Ni}$ into even heavier trans-iron group elements. Unfortunately, we are presently not able to search for these elements because of the lack of reliable atomic data of the expected high ionization stages.

Acknowledgments. M.Z. thanks the Astronomische Gesellschaft for a travel grant. T.R. is supported by the German Astrophysical Virtual Observatory (GAVO) project of the German Federal Ministry of Education and Research (BMBF) under grant 05 AC6VTB. J.W.K. is supported by the FUSE project, funded by NASA contract NAS5-32985.

\footnotetext{
$1_{\text {http://vo.ari.uni-heidelberg.de/ssatr-0.01/TrSpectra.jsp? }}$

${ }^{2}$ German Astrophysical Virtual Observatory
} 


\section{References}

Dreizler, S., Werner, K., \& Heber, U. 1997, in "Planetary nebulae", eds. H. J. Habing and H. J. G. L. M. Lamers, IAU Symposium, 180, 103

Herwig, F. 2001, Ap\&SS, 275, 15

Miksa, S., Deetjen, J. L., Dreizler, S., et al. 2002, A\&A, 389, 953

Napiwotzki, R., Christlieb, N., Drechsel, H., et al. 2001, AN, 322, 411

Rauch, T., \& Deetjen, J. L. 2003, in "Stellar Atmosphere Modeling", eds. I. Hubeny, D. Mihalas, and K. Werner, ASP Conf. Ser., 288, 103

Werner, K., Deetjen, J. L., Dreizler, S., et al. 2003, in "Stellar Atmosphere Modeling", eds. I. Hubeny, D. Mihalas, and K. Werner, ASP Conf. Ser., 288, 31

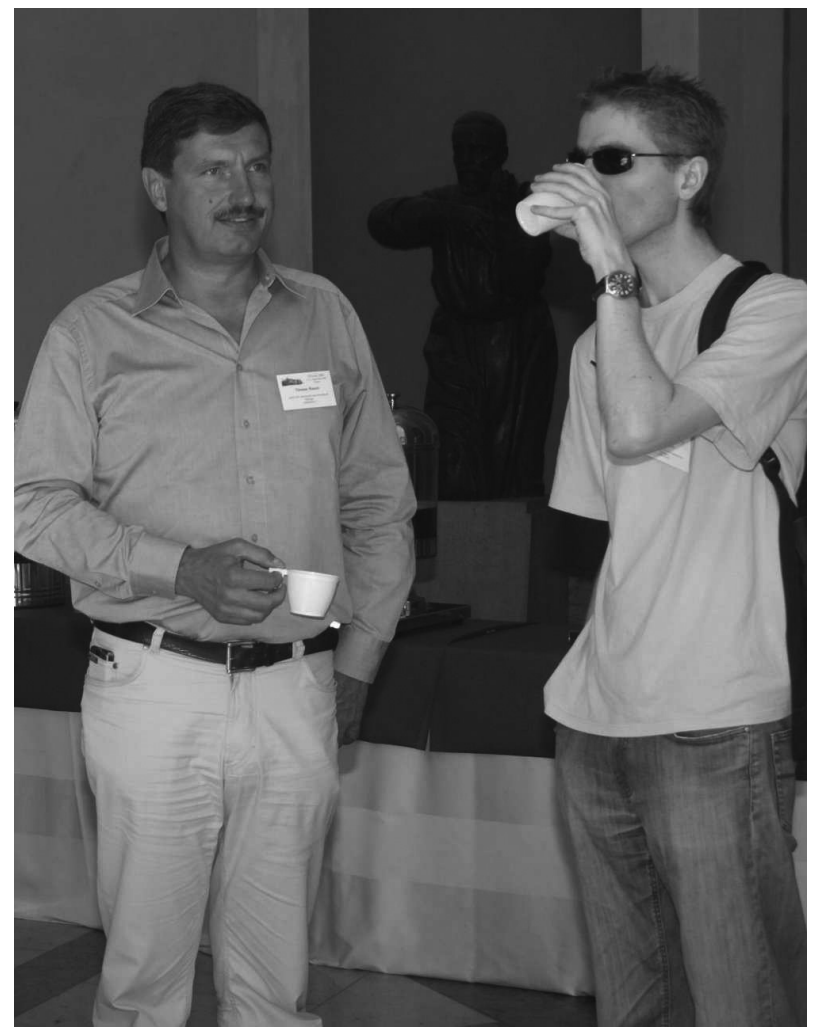



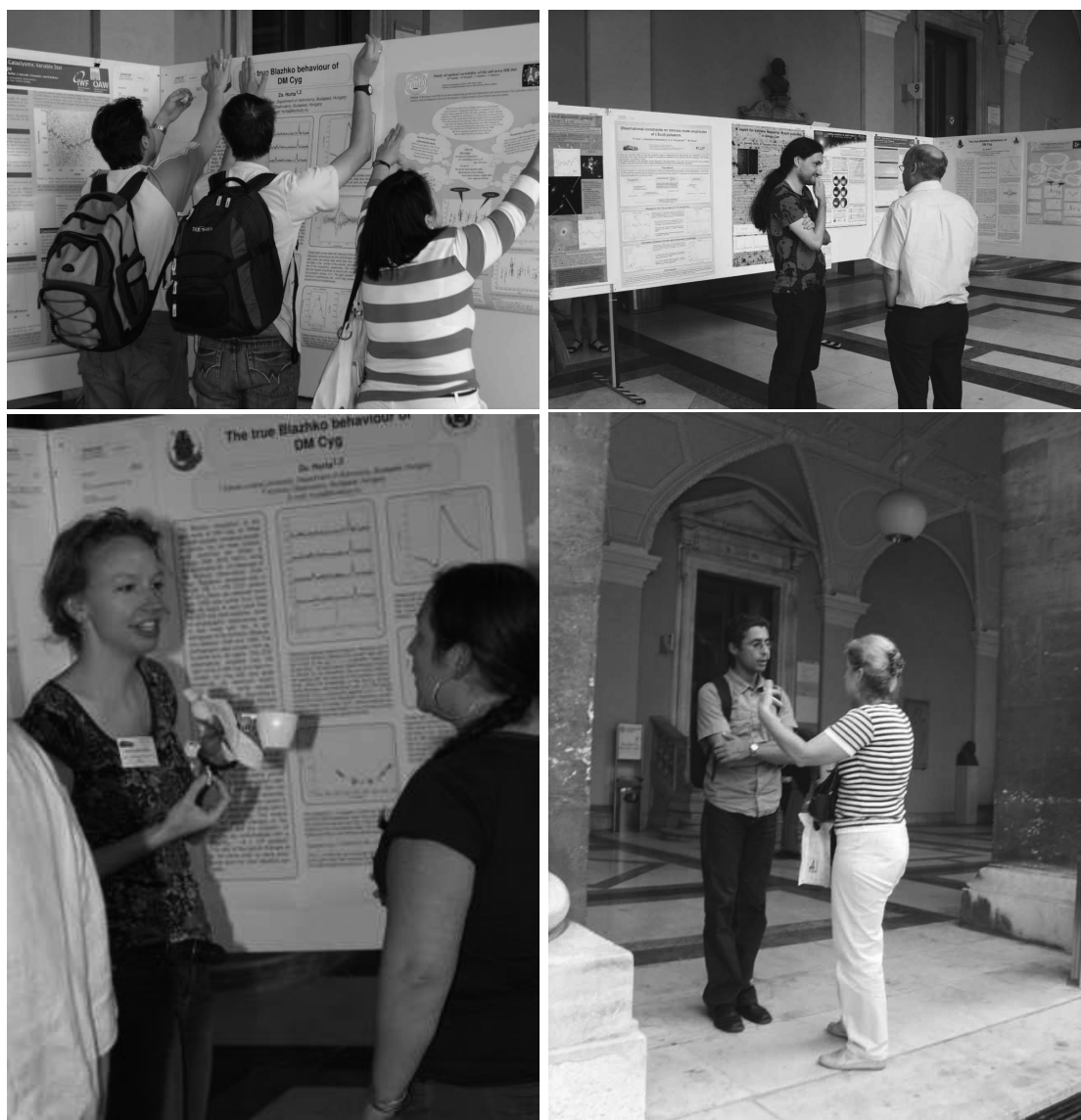

Poster session and discussions in the Arcades. 